\title{
A Metric Observer for Induction Motors Control
}

\author{
Mohamed Benbouzid, ${ }^{1,2}$ Abdelkrim Benchaib, ${ }^{3}$ Gang Yao, ${ }^{2}$ \\ Brice Beltran, ${ }^{1,4}$ and Olivier Chocron ${ }^{5}$ \\ ${ }^{1}$ University of Brest, FRE CNRS 3744 IRDL, 29238 Brest, France \\ ${ }^{2}$ Shanghai Maritime University, Shanghai 201306, China \\ ${ }^{3}$ Alstom Grid, 91300 Massy, France \\ ${ }^{4} D G A, 40000$ Mont-de-Marsan, France \\ ${ }^{5}$ ENI Brest, FRE CNRS 3744 IRDL, 29280 Plouzané, France \\ Correspondence should be addressed to Mohamed Benbouzid; mohamed.benbouzid@univ-brest.fr
}

Received 14 November 2015; Revised 23 March 2016; Accepted 11 April 2016

Academic Editor: Kalyana C. Veluvolu

Copyright ( $) 2016$ Mohamed Benbouzid et al. This is an open access article distributed under the Creative Commons Attribution License, which permits unrestricted use, distribution, and reproduction in any medium, provided the original work is properly cited.

This paper deals with metric observer application for induction motors. Firstly, assuming that stator currents and speed are measured, a metric observer is designed to estimate the rotor fluxes. Secondly, assuming that only stator currents are measured, another metric observer is derived to estimate rotor fluxes and speed. The proposed observer validity is checked throughout simulations on a $4 \mathrm{~kW}$ induction motor drive.

\section{Introduction}

In the two last decades, the most significant developments in induction motors control have been field-oriented control [1] and nonlinear input-output and state feedback linearization techniques [2] with real-world industry application. More advanced control techniques have also been proposed, such as (1) passivity-based approach, which exploits the system energy dissipation property to solve the underlying control problem [3], (2) sliding mode-based control approaches [4$7]$ and the higher-order ones [8,9], and (3) flatness-based control approaches [10].

Otherwise, in most of the above-mentioned control approaches, an observer has to be used since a part of the motor state is not measurable in industrial applications. Several observers have been proposed in the literature. The most well-known and popular ones are given in [11, 12], in which authors have proposed the model reference adaptive system (MRAS) for the estimation of the induction motor speed, from measured phase voltages and currents, based on the adaptive control theory. Some other observer has been proposed based on the context of more advanced and/or intelligent control technique such as sliding mode [13], high gain observer [14], and the mean value theorem [15].
Screening deeply the literature on observers design for nonlinear systems, specific ones have been proposed in [16, 17], namely, metric observers. In this particular and still challenging observer design context, it is proposed to investigate the effectiveness of metric observers for induction motors control. This paper's objective is therefore twofold: (1) assuming the stator currents and rotor speed to be measured, a reduced-order metric observer is proposed to estimate the induction motor rotor fluxes; (2) assuming that only the stator currents are measured, a nonlinear reduced-order metric observer is derived to estimate rotor fluxes and speed.

The paper is organized as follows. Section 2 deals with the induction modeling. In the first part of Section 3, a reducedorder metric observer is derived to estimate the rotor flux and, in the second part, a nonlinear reduced-order one for rotor flux and rotor speed estimation is proposed. Section 4 gives simulation results for validation purposes. Finally some concluding remarks end the paper.

\section{Induction Motor Model}

Assuming that we have balanced three-phase AC voltages and that stator windings are uniformly distributed, and based 
on the well-known two-phase equivalent machine representation, the induction motor can be described by fifth-order nonlinear differential equations with four electrical variables (currents and fluxes), one mechanical variable (rotor speed), and two control variables (stator voltages). In a fixed $(a, b)$ frame, one has

$$
\begin{gathered}
\dot{X}=f(X, U)=A(\omega(t)) X+B U, \\
\dot{\omega}(t)=k\left(\varphi_{a} i_{b}-\varphi_{b} i_{a}\right)-\frac{T_{L}}{J}-\frac{f_{\mathrm{rot}}}{J} \omega,
\end{gathered}
$$

where

$$
\begin{aligned}
U & =\left[\begin{array}{ll}
u_{a} & u_{b}
\end{array}\right]^{T}, \\
X & =\left[\begin{array}{llll}
x_{1} & x_{2} & x_{3} & x_{4}
\end{array}\right]^{T}=\left[\begin{array}{llll}
i_{a} & i_{b} & \varphi_{a} & \varphi_{b}
\end{array}\right]^{T}, \\
A(\omega(t)) & =\left(\begin{array}{cccc}
-\gamma & 0 & \frac{K}{T_{r}} & p K \omega(t) \\
0 & -\gamma & -p K \omega(t) & \frac{K}{T_{r}} \\
\frac{M}{T_{r}} & 0 & -\frac{1}{T_{r}} & -p \omega(t) \\
0 & \frac{M}{T_{r}} & p \omega(t) & -\frac{1}{T_{r}}
\end{array}\right), \\
B & =\left(\begin{array}{cc}
\frac{1}{\sigma L_{s}} & 0 \\
0 & \frac{1}{\sigma L_{s}} \\
0 & 0 \\
0 & 0
\end{array}\right)
\end{aligned}
$$

with

$$
\begin{aligned}
\sigma & =1-\frac{M^{2}}{L_{s} L_{r}}, \\
K & =\frac{M}{\sigma L_{s} L_{r}}, \\
\gamma & =\frac{R_{s}}{\sigma L_{s}}+\frac{R_{r} M^{2}}{\sigma L_{s} L_{r}^{2}}, \\
k & =p \frac{M}{J L_{r}} .
\end{aligned}
$$

It is worth noticing that the only measured variables are stator currents so that the output state equation is

$$
Y=H(X)=\left(\begin{array}{llll}
1 & 0 & 0 & 0 \\
0 & 1 & 0 & 0
\end{array}\right) X .
$$

It can be easily shown that state $X$ is observable from $Y$.

\section{The Metric Observer}

In this section, we propose a reduced-order metric observer for rotor fluxes estimation. Then, we will introduce a nonlinear reduced-order one for rotor fluxes and rotor speed estimations.
3.1. Reduced-Order Observer for Fluxes Estimation. Assuming that the stator currents $x_{1}$ and $x_{2}$ and the rotor speed $\omega$ are measured, we consider the induction motor fourth-order model. Consider

$$
\begin{aligned}
& \dot{x}_{1}=-\gamma x_{1}+\frac{K}{T_{r}} x_{3}+p K \omega(t) x_{4}+\frac{1}{\sigma L_{s}} u_{a}, \\
& \dot{x}_{2}=-\gamma x_{2}+\frac{K}{T_{r}} x_{4}-p K \omega(t) x_{3}+\frac{1}{\sigma L_{s}} u_{b}, \\
& \dot{x}_{3}=\frac{M}{T_{r}} x_{1}-\frac{1}{T_{r}} x_{3}-p \omega(t) x_{4}, \\
& \dot{x}_{4}=\frac{M}{T_{r}} x_{2}-\frac{1}{T_{r}} x_{4}+p \omega(t) x_{3} .
\end{aligned}
$$

A possible reduced-order observer for $\widehat{x}_{3}$ and $\widehat{x}_{4}$ is a simple copy of the two last equations of our dynamic model. Consider

$$
\begin{aligned}
& \dot{\hat{x}}_{3}=\frac{M}{T_{r}} \widehat{x}_{1}-\frac{1}{T_{r}} \widehat{x}_{3}-p \omega(t) \hat{x}_{4}, \\
& \dot{\hat{x}}_{4}=\frac{M}{T_{r}} \widehat{x}_{2}-\frac{1}{T_{r}} \widehat{x}_{4}+p \omega(t) \widehat{x}_{3} .
\end{aligned}
$$

To ensure this observer's exponential convergence, we introduce two intermediate variables [16]:

$$
\begin{aligned}
& \bar{x}_{3}=\widehat{x}_{3}+\Gamma \widehat{x}_{1}, \\
& \bar{x}_{4}=\widehat{x}_{4}+\Gamma \widehat{x}_{2} .
\end{aligned}
$$

The new dynamic equations are given by

$$
\begin{aligned}
& \dot{\bar{x}}_{3}=(\Gamma K-1)\left(\frac{\bar{x}_{3}}{T_{r}}+p \omega \bar{x}_{4}\right)+f_{1}, \\
& \dot{\bar{x}}_{4}=(1-\Gamma K)\left(p \omega \bar{x}_{3}-\frac{1}{T_{r}} \bar{x}_{4}\right)+f_{2}
\end{aligned}
$$

with

$$
\begin{aligned}
f_{1}= & {\left[\left(\frac{M}{T_{r}}-\gamma \Gamma\right)+\frac{\Gamma}{T_{r}}(1-\Gamma K)\right] \widehat{x}_{1} } \\
& -(\Gamma K-1) \Gamma p \omega \widehat{x}_{2}+\frac{\Gamma}{\sigma L_{s}} u_{a}, \\
f_{2}= & (\Gamma K-1) \Gamma p \omega \widehat{x}_{1} \\
& +\left[\left(\frac{M}{T_{r}}-\gamma \Gamma\right)+\frac{\Gamma}{T_{r}}(1-\Gamma K)\right] \widehat{x}_{2}+\frac{\Gamma}{\sigma L_{s}} u_{b} .
\end{aligned}
$$

$\widehat{x}_{1}$ and $\hat{x}_{2}$ are now replaced by measurements $x_{1}$ and $x_{2}$ [16]. This leads to the following observer equation with the intermediate variables:

$$
\begin{gathered}
\dot{\bar{x}}_{3}=(\Gamma K-1)\left(\frac{1}{T_{r}} \bar{x}_{3}+p \omega \bar{x}_{4}\right)+h_{1}, \\
\dot{\bar{x}}=(1-\Gamma K)\left(p \omega \bar{x}_{3}-\frac{1}{T_{r}} \bar{x}_{4}\right)+h_{2}
\end{gathered}
$$


with

$$
\begin{aligned}
h_{1}= & {\left[\left(\frac{M}{T_{r}}-\gamma \Gamma\right)+\frac{\Gamma}{T_{r}}(1-\Gamma K)\right] x_{1} } \\
& -(\Gamma K-1) \Gamma p \omega x_{2}+\frac{\Gamma}{\sigma L_{s}} u_{a}, \\
h_{2}= & (\Gamma K-1) \Gamma p \omega x_{1} \\
& +\left[\left(\frac{M}{T_{r}}-\gamma \Gamma\right)+\frac{\Gamma}{T_{r}}(1-\Gamma K)\right] x_{2}+\frac{\Gamma}{\sigma L_{s}} u_{b} .
\end{aligned}
$$

The result of this computation is then mapped back to the original reduced state space with

$$
\begin{aligned}
& \widehat{x}_{3}=\bar{x}_{3}-\Gamma x_{1}, \\
& \widehat{x}_{4}=\bar{x}_{4}-\Gamma x_{2} .
\end{aligned}
$$

This leads to the following new observer dynamics in $\widehat{x}_{3}$ and $\widehat{x}_{4}[16]$ :

$$
\begin{aligned}
& \dot{\hat{x}}_{3}=(\Gamma K-1)\left(\frac{1}{T_{r}} \widehat{x}_{3}+p \omega \widehat{x}_{4}\right)+g_{1}, \\
& \dot{\hat{x}}_{4}=(1-\Gamma K)\left(p \omega \widehat{x}_{3}-\frac{1}{T_{r}} \widehat{x}_{4}\right)+g_{2}
\end{aligned}
$$

with

$$
\begin{aligned}
& g_{1}=\frac{M}{T_{r}} x_{1}-\frac{\Gamma K}{T_{r}} \widehat{x}_{3}-p \omega \Gamma K \widehat{x}_{4}, \\
& g_{2}=\frac{M}{T_{r}} x_{2}-\frac{\Gamma K}{T_{r}} \widehat{x}_{4}+p \omega \Gamma K \widehat{x}_{3} .
\end{aligned}
$$
is

The Jacobian or rate of deformation tensor of this system

$$
F=\left(\begin{array}{cc}
(\Gamma K-1) \frac{1}{T_{r}} & (\Gamma K-1) p \omega \\
(1-\Gamma K) p \omega & (\Gamma K-1) \frac{1}{T_{r}}
\end{array}\right)
$$

The strain tensor rate is

$$
E=\left(\begin{array}{cc}
\frac{2}{T_{r}}(\Gamma K-1) & 0 \\
0 & \frac{2}{T_{r}}(\Gamma K-1)
\end{array}\right) .
$$

We follow the same procedure as in $[16,17]$. The exponential convergence of the reduced-order observer is guaranteed for a strain tensor uniformly negative definite rate. The rate of the strain tensor is uniformly negative definite if and only if $\exists \beta>0$ such that the following conditions are satisfied:

$$
\begin{gathered}
\frac{2}{T_{r}}(\Gamma K-1) \leq-\beta<0, \\
{\left[\frac{2}{T_{r}}(\Gamma K-1)\right]^{2} \geq \beta>0 .}
\end{gathered}
$$

3.2. Reduced-Order Observer for Rotor Fluxes and Speed Estimation. Assuming now that just the stator currents are available, we will design a reduced-order observer to estimate rotor fluxes and speed. Consider the fifth-order model written as

$$
\begin{aligned}
& \dot{x}_{1}=-\gamma x_{1}+\frac{K}{T_{r}} x_{3}+p K \omega x_{4}+\frac{1}{\sigma L_{s}} u_{a}, \\
& \dot{x}_{2}=-\gamma x_{2}+\frac{K}{T_{r}} x_{4}-p K \omega x_{3}+\frac{1}{\sigma L_{s}} u_{b}, \\
& \dot{x}_{3}=\frac{M}{T_{r}} x_{1}-\frac{1}{T_{r}} x_{3}-p x_{4} \omega, \\
& \dot{x}_{4}=\frac{M}{T_{r}} x_{2}-\frac{1}{T_{r}} x_{4}+p x_{3} \omega, \\
& \dot{\omega}=k\left(x_{2} x_{3}-x_{1} x_{4}\right)-\frac{f_{\text {rot }}}{J} \omega-\frac{T_{L}}{J} .
\end{aligned}
$$

A possible reduced-order observer for $x_{3}, x_{4}$, and $\omega$ is a simple copy of the last three equations of our fifth-order dynamic model:

$$
\begin{aligned}
& \dot{\hat{x}}_{3}=\frac{M}{T_{r}} \widehat{x}_{1}-\frac{1}{T_{r}} \widehat{x}_{3}-p \widehat{x}_{4} \widehat{\omega}, \\
& \dot{\hat{x}}_{4}=\frac{M}{T_{r}} \widehat{x}_{2}-\frac{1}{T_{r}} \widehat{x}_{4}+p \widehat{x}_{3} \widehat{\omega} \\
& \dot{\hat{\omega}}=\operatorname{cst}\left(\hat{x}_{2} \widehat{x}_{3}-\widehat{x}_{1} \widehat{x}_{4}\right)-\frac{f_{\text {rot }}}{J} \widehat{\omega}-\frac{T_{L}}{J} .
\end{aligned}
$$

To ensure the above observer's exponential convergence, we introduce three intermediate variables:

$$
\begin{aligned}
& \bar{x}_{3}=\widehat{x}_{3}+\widehat{\Phi}_{1}, \\
& \bar{x}_{4}=\widehat{x}_{4}+\widehat{\Phi}_{2}, \\
& \bar{\omega}=\widehat{\omega}+\widehat{\Phi}_{3}
\end{aligned}
$$

with

$$
\begin{gathered}
\widehat{\Phi}_{1}=\Gamma_{1}\left[\begin{array}{l}
\widehat{x}_{1} \\
\widehat{x}_{2}
\end{array}\right], \\
\widehat{\Phi}_{2}=\Gamma_{2}\left[\begin{array}{l}
\widehat{x}_{1} \\
\widehat{x}_{2}
\end{array}\right], \\
\widehat{\Phi}_{3}=\Gamma_{3}\left[\begin{array}{l}
\widehat{x}_{1} \\
\widehat{x}_{2}
\end{array}\right], \\
\Gamma_{i}=\left[\begin{array}{ll}
\Gamma_{i 1} & \Gamma_{i 2}
\end{array}\right] .
\end{gathered}
$$


The coordinates' intermediate change leads to the following new observer equations:

$$
\begin{aligned}
& \dot{\bar{x}}_{3}=\left[-\frac{1}{T_{r}}+\Gamma_{11} \frac{K}{T_{r}}-\Gamma_{12} p K \widehat{\Phi}_{3}\right] \bar{x}_{3} \\
& +\left[\Gamma_{12} \frac{K}{T_{r}}-p\left(\Gamma_{11} K-1\right) \widehat{\Phi}_{3}\right] \bar{x}_{4} \\
& +\left[\Gamma_{12} p K \bar{x}_{3}+p\left(\Gamma_{11} K-1\right) \bar{x}_{4}+\widehat{J}_{1}\right] \bar{\omega}+\widehat{G}_{1}, \\
& \widehat{G}_{1}=\widehat{F}_{1}-\frac{1}{T_{r}}\left(\Gamma_{11} K-1\right) \widehat{\Phi}_{1}-\Gamma_{12} \frac{K}{T_{r}} \widehat{\Phi}_{2}-\widehat{J}_{1} \widehat{\Phi}_{3}, \\
& \widehat{F}_{1}=\left(\frac{M}{T_{r}}-\gamma \Gamma_{11}\right) \widehat{x}_{1}-\gamma \Gamma_{12} \widehat{x}_{2}+\frac{\Gamma_{11}}{\sigma L_{s}} u_{a}+\frac{\Gamma_{12}}{\sigma L_{s}} u_{b} \text {, } \\
& \widehat{J}_{1}=\Gamma_{12} p K \widehat{\Phi}_{1}-p\left(\Gamma_{11} K-1\right) \widehat{\Phi}_{2} \text {, } \\
& \dot{\bar{x}}_{4}=\left[\Gamma_{21} \frac{K}{T_{r}}+p\left(\Gamma_{22} K-1\right) \widehat{\Phi}_{3}\right] \bar{x}_{3} \\
& +\left[-\frac{1}{T_{r}}+\Gamma_{22} \frac{K}{T_{r}}-\Gamma_{21} p K \widehat{\Phi}_{3}\right] \bar{x}_{4} \\
& +\left[p\left(1-\Gamma_{22} K\right) \bar{x}_{3}+\Gamma_{21} p K \bar{x}_{4}+\widehat{J}_{2}\right] \bar{\omega}+\widehat{G}_{2} \text {, } \\
& \widehat{G}_{2}=\widehat{F}_{2}-\frac{1}{T_{r}}\left(\Gamma_{22} K-1\right) \widehat{\Phi}_{2}-\Gamma_{21} \frac{K}{T_{r}} \widehat{\Phi}_{1}-\widehat{J}_{2} \widehat{\Phi}_{3} \text {, } \\
& \widehat{F}_{1}=-\gamma \Gamma_{21} \widehat{x}_{1}+\left(\frac{M}{T_{r}}-\gamma \Gamma_{22}\right) \widehat{x}_{2}+\frac{\Gamma_{21}}{\sigma L_{s}} u_{a}+\frac{\Gamma_{22}}{\sigma L_{s}} u_{b} \text {, } \\
& \widehat{J}_{2}=p\left(\Gamma_{22} K-1\right) \widehat{\Phi}_{1}+\Gamma_{21} p K \widehat{\Phi}_{2} \text {, } \\
& \dot{\bar{\omega}}=\left[\operatorname{cst} \widehat{x}_{2}+\Gamma_{31} \frac{K}{T_{r}}+p K \Gamma_{32} \widehat{\Phi}_{3}\right] \bar{x}_{3} \\
& +\left[-\operatorname{cst} \widehat{x}_{1}+\Gamma_{32} \frac{K}{T_{r}}-p K \Gamma_{31} \widehat{\Phi}_{3}\right] \bar{x}_{4} \\
& +\left[-p K \Gamma_{32} \bar{x}_{3}+p K \Gamma_{32} \bar{x}_{4}+\widehat{J}_{3}\right] \bar{\omega}+\widehat{G}_{3}, \\
& \widehat{G}_{3}=\widehat{F}_{3}-\left(\operatorname{cst} \widehat{x}_{2}+\Gamma_{31} \frac{K}{T_{r}}\right) \widehat{\Phi}_{1} \\
& -\left(-\operatorname{cst} \widehat{x}_{1}+\Gamma_{32} \frac{K}{T_{r}}\right) \widehat{\Phi}_{2}-\widehat{J}_{3} \widehat{\Phi}_{3} \text {, } \\
& \widehat{F}_{3}=-\gamma \Gamma_{31} \widehat{x}_{1}-\gamma \Gamma_{32} \widehat{x}_{2}+\frac{\Gamma_{31}}{\sigma L_{s}} u_{a}+\frac{\Gamma_{32}}{\sigma L_{s}} u_{b}-\frac{T_{L}}{J}, \\
& \widehat{J}_{3}=p K \Gamma_{32} \widehat{\Phi}_{1}-p K \Gamma_{31} \widehat{\Phi}_{2}-\frac{f_{\mathrm{rot}}}{J} \text {. }
\end{aligned}
$$

$\widehat{x}_{1}$ and $\hat{x}_{2}$ are now replaced by measurements $x_{1}$ and $x_{2}$. This leads to the following observer equation with the intermediate variables:

$$
\begin{aligned}
\dot{\bar{x}}_{3}= & {\left[-\frac{1}{T_{r}}+\Gamma_{11} \frac{K}{T_{r}}+\Gamma_{12} p K \Phi_{3}\right] \bar{x}_{3} } \\
& +\left[\Gamma_{12} \frac{K}{T_{r}}-p\left(\Gamma_{11} K-1\right) \Phi_{3}\right] \bar{x}_{4}
\end{aligned}
$$

$$
\begin{aligned}
& +\left[-\Gamma_{12} p K \bar{x}_{3}+p\left(\Gamma_{11} K-1\right) \bar{x}_{4}+J_{1}\right] \bar{\omega} \\
& +G_{1} \text {, } \\
& G_{1}=F_{1}-\frac{1}{T_{r}}\left(\Gamma_{11} K-1\right) \Phi_{1}-\Gamma_{12} \frac{K}{T_{r}} \Phi_{2}-J_{1} \Phi_{3}, \\
& F_{1}=\left(\frac{M}{T_{r}}-\gamma \Gamma_{11}\right) x_{1}-\gamma \Gamma_{12} x_{2}+\frac{\Gamma_{11}}{\sigma L_{s}} u_{a}+\frac{\Gamma_{12}}{\sigma L_{s}} u_{b}, \\
& J_{1}=\Gamma_{12} p K \Phi_{1}-p\left(\Gamma_{11} K-1\right) \Phi_{2} \text {, } \\
& \dot{\bar{x}}_{4}=\left[\Gamma_{21} \frac{K}{T_{r}}+p\left(\Gamma_{22} K-1\right) \Phi_{3}\right] \bar{x}_{3} \\
& +\left[-\frac{1}{T_{r}}+\Gamma_{22} \frac{K}{T_{r}}-\Gamma_{21} p K \Phi_{3}\right] \bar{x}_{4} \\
& +\left[p\left(1-\Gamma_{22} K\right) \bar{x}_{3}+\Gamma_{21} p K \bar{x}_{4}+J_{2}\right] \bar{\omega}+G_{2} \text {, } \\
& G_{2}=F_{2}-\frac{1}{T_{r}}\left(\Gamma_{22} K-1\right) \Phi_{2}-\Gamma_{21} \frac{K}{T_{r}} \Phi_{1}-J_{2} \Phi_{3} \text {, } \\
& F_{1}=-\gamma \Gamma_{21} x_{1}+\left(\frac{M}{T_{r}}-\gamma \Gamma_{22}\right) x_{2}+\frac{\Gamma_{21}}{\sigma L_{s}} u_{a}+\frac{\Gamma_{22}}{\sigma L_{s}} u_{b} \text {, } \\
& J_{2}=p\left(\Gamma_{22} K-1\right) \Phi_{1}+\Gamma_{21} p K \Phi_{2} \text {, } \\
& \dot{\bar{\omega}}=\left[\operatorname{cst} x_{2}+\Gamma_{31} \frac{K}{T_{r}}+p K \Gamma_{32} \Phi_{3}\right] \bar{x}_{3} \\
& +\left[-\operatorname{cst} x_{1}+\Gamma_{32} \frac{K}{T_{r}}-p K \Gamma_{31} \Phi_{3}\right] \bar{x}_{4} \\
& +\left[-p K \Gamma_{32} \bar{x}_{3}+p K \Gamma_{32} \bar{x}_{4}+J_{3}\right] \bar{\omega}+G_{3}, \\
& G_{3}=F_{3}-\left(\operatorname{cst} x_{2}+\Gamma_{31} \frac{K}{T_{r}}\right) \Phi_{1} \\
& -\left(-\operatorname{cst} x_{1}+\Gamma_{32} \frac{K}{T_{r}}\right) \Phi_{2}-J_{3} \Phi_{3} \text {, } \\
& F_{3}=-\gamma \Gamma_{31} x_{1}-\gamma \Gamma_{32} x_{2}+\frac{\Gamma_{31}}{\sigma L_{s}} u_{a}+\frac{\Gamma_{32}}{\sigma L_{s}} u_{b}-\frac{T_{L}}{J}, \\
& J_{3}=p K \Gamma_{32} \Phi_{1}-p K \Gamma_{31} \Phi_{2}-\frac{f_{\text {rot }}}{J}
\end{aligned}
$$

with

$$
\begin{aligned}
& \Phi_{1}=\Gamma_{1}\left[\begin{array}{l}
x_{1} \\
x_{2}
\end{array}\right], \\
& \Phi_{2}=\Gamma_{2}\left[\begin{array}{l}
x_{1} \\
x_{2}
\end{array}\right], \\
& \Phi_{3}=\Gamma_{3}\left[\begin{array}{l}
x_{1} \\
x_{2}
\end{array}\right] .
\end{aligned}
$$


The result of this computation is then mapped back to the original reduced state space with

$$
\begin{aligned}
& \widehat{x}_{3}=\bar{x}_{3}-\Phi_{1}, \\
& \widehat{x}_{4}=\bar{x}_{4}-\Phi_{2}, \\
& \widehat{\omega}=\bar{\omega}-\Phi_{3} .
\end{aligned}
$$

As discussed in $[16,17]$, this leads to the following new observer dynamics in $\widehat{x}_{3}, \widehat{x}_{4}$, and $\widehat{\omega}$. The estimated flux $\widehat{x}_{3}=$ $\widehat{\varphi}_{a}$ is given by

$$
\begin{aligned}
\dot{\hat{x}}_{3}= & \frac{1}{T_{r}}\left(\Gamma_{11} K-1\right) \hat{x}_{3}+\Gamma_{12} \frac{K}{T_{r}} \widehat{x}_{4} \\
& +\left(-\Gamma_{12} p K \hat{x}_{3}+p\left(\Gamma_{11} K-1\right) \hat{x}_{4}\right) \widehat{\omega}+H_{1}
\end{aligned}
$$

with

$$
\begin{aligned}
H_{1}= & A_{1} \Phi_{1}+A_{2} \Phi_{2}+G_{1} \\
& -\Gamma_{11}\left(-\gamma x_{1}+\frac{K}{T_{r}} x_{3}+p K \omega x_{4}+\frac{1}{\sigma L_{s}} u_{a}\right) \\
& -\Gamma_{12}\left(-\gamma x_{2}+\frac{K}{T_{r}} x_{4}-p K \omega x_{3}+\frac{1}{\sigma L_{s}} u_{b}\right), \\
A_{1}= & -\frac{1}{T_{r}}+\Gamma_{11} \frac{K}{T_{r}}-\Gamma_{12} p K \Phi_{3}, \\
A_{2}= & \Gamma_{12} \frac{K}{T_{r}}-p\left(\Gamma_{11} K-1\right) \Phi_{3} .
\end{aligned}
$$

The estimated flux $\widehat{x}_{4}=\widehat{\varphi}_{b}$ is given by

$$
\begin{aligned}
\dot{\hat{x}}_{4}= & \Gamma_{21} \frac{K}{T_{r}} \widehat{x}_{3}+\left[-\frac{1}{T_{r}}+\Gamma_{22} \frac{K}{T_{r}}\right] \widehat{x}_{4} \\
& +\left[p\left(1-\Gamma_{22} K\right) \hat{x}_{3}+\Gamma_{21} p K \hat{x}_{4}\right] \widehat{\omega}+H_{2}
\end{aligned}
$$

with

$$
\begin{aligned}
H_{2}= & B_{1} \Phi_{1}+B_{2} \Phi_{2}+G_{1} \\
& -\Gamma_{21}\left(-\gamma x_{1}+\frac{K}{T_{r}} x_{3}+p K \omega x_{4}+\frac{1}{\sigma L_{s}} u_{a}\right) \\
& -\Gamma_{22}\left(-\gamma x_{2}+\frac{K}{T_{r}} x_{4}-p K \omega x_{3}+\frac{1}{\sigma L_{s}} u_{b}\right), \\
B_{1}= & \Gamma_{21} \frac{K}{T_{r}}+p\left(\Gamma_{22} K-1\right) \Phi_{3}, \\
B_{2}= & -\frac{1}{T_{r}}+\Gamma_{22} \frac{K}{T_{r}}-\Gamma_{21} p K \Phi_{3} .
\end{aligned}
$$

The estimated speed $\widehat{x}_{5}=\widehat{\omega}$ is given by

$$
\begin{aligned}
\dot{\hat{\omega}}= & {\left[\operatorname{cst} x_{2}+\Gamma_{31} \frac{K}{T_{r}}\right] \widehat{x}_{3}+\left[-\operatorname{cst} x_{1}+\Gamma_{32} \frac{K}{T_{r}}\right] \widehat{x}_{4} } \\
& +\left[-p K \Gamma_{32} \widehat{x}_{3}+p K \Gamma_{32} \widehat{x}_{4}\right] \widehat{\omega}+H_{3}
\end{aligned}
$$

with

$$
\begin{aligned}
H_{3}= & C_{1} \Phi_{1}+C_{2} \Phi_{2}+G_{3} \\
& -\Gamma_{31}\left(-\gamma x_{1}+\frac{K}{T_{r}} x_{3}+p K \omega x_{4}+\frac{1}{\sigma L_{s}} u_{a}\right) \\
& -\Gamma_{32}\left(-\gamma x_{2}+\frac{K}{T_{r}} x_{4}-p K \omega x_{3}+\frac{1}{\sigma L_{s}} u_{b}\right), \\
C_{1}= & \operatorname{cst} x_{2}+\Gamma_{31} \frac{K}{T_{r}}+p K \Gamma_{32} \Phi_{3}, \\
C_{2}= & -\operatorname{cst} x_{1}+\Gamma_{32} \frac{K}{T_{r}}-p K \Gamma_{31} \Phi_{3} .
\end{aligned}
$$

is

The Jacobian or rate of deformation tensor of this system

$$
F=\left(\begin{array}{lll}
F_{11} & F_{12} & F_{13} \\
F_{21} & F_{22} & F_{23} \\
F_{31} & F_{32} & F_{33}
\end{array}\right)
$$

with

$$
\begin{aligned}
& F_{11}=-\frac{1}{T_{r}}+\Gamma_{11} \frac{K}{T_{r}}-\Gamma_{12} p K \widehat{\omega}, \\
& F_{12}=\Gamma_{12} \frac{K}{T_{r}}+p\left(\Gamma_{11} K-1\right) \widehat{\omega}, \\
& F_{13}=-\Gamma_{12} p K \widehat{x}_{3}+p\left(\Gamma_{11} K-1\right) \widehat{x}_{4}, \\
& F_{21}=\Gamma_{21} \frac{K}{T_{r}}-p\left(\Gamma_{22} K-1\right) \widehat{\omega}, \\
& F_{22}=-\frac{1}{T_{r}}+\Gamma_{22} \frac{K}{T_{r}}+\Gamma_{21} p K \widehat{\omega}, \\
& F_{23}=p\left(1-\Gamma_{22} K\right) \hat{x}_{3}+\Gamma_{21} p K \widehat{x}_{4}, \\
& F_{31}=\operatorname{cst} x_{2}+\Gamma_{31} \frac{K}{T_{r}}-p K \Gamma_{32} \widehat{\omega}, \\
& F_{32}=-\operatorname{cst} x_{1}+\Gamma_{32} \frac{K}{T_{r}}+p K \Gamma_{31} \widehat{\omega}, \\
& F_{33}=-p K \Gamma_{32} \widehat{x}_{3}+p K \Gamma_{32} \widehat{x}_{4} .
\end{aligned}
$$

The strain tensor rate is

$$
E=\left(\begin{array}{lll}
E_{11} & E_{12} & E_{13} \\
E_{21} & E_{22} & E_{23} \\
E_{31} & E_{32} & E_{33}
\end{array}\right)
$$


with

$$
\begin{aligned}
& E_{11}=2 F_{11}, \\
& E_{12}=E_{21}=F_{12}+F_{21}, \\
& E_{13}=E_{31}=F_{13}+F_{31}, \\
& E_{22}=2 F_{22}, \\
& E_{23}=E_{32}=F_{23}+F_{32}, \\
& E_{33}=2 F_{33} .
\end{aligned}
$$

Exponential convergence of the reduced-order observer is guaranteed for a uniformly negative definite rate of strain tensor. The strain tensor rate is uniformly negative definite if and only if $\exists \beta>0$ such that the following conditions are satisfied:

$$
\begin{aligned}
E_{11} & \leq-\beta<0, \\
\operatorname{det}\left(\begin{array}{ll}
E_{11} & E_{12} \\
E_{21} & E_{22}
\end{array}\right) & \geq \beta>0, \\
\operatorname{det}(E) & \leq-\beta<0 .
\end{aligned}
$$

\section{Simulations Results}

This section deals with simulations highlighting the proposed reduced-order metric observer's feasibility. The simulated induction motor ratings are the following: $4 \mathrm{~kW}, 4$ poles, $1440 \mathrm{rpm}, R_{s}=1.9 \Omega, R_{r}=1.73 \Omega, L_{s}=0.1157 \mathrm{H}, L_{r}=$ $0.1154 \mathrm{H}, L_{m}=0.1126 \mathrm{H}$, and $J=0.041 \mathrm{Kg} \cdot \mathrm{N} / \mathrm{m}^{2}$.

4.1. Simulation Results for the Reduced-Order Observer for Fluxes Estimation. Figures 1-3 show the real flux, its estimation, and the estimation error in $a$-axis $\left(\varphi_{a}-\widehat{\varphi}_{a}\right)$, respectively. These results were obtained for an unloaded machine $\left(T_{L}=\right.$ 0 ), with an observer gain of $\Gamma=0.05$.

Figures 4-6 illustrate the real flux, its estimation, and the estimation error in $b$-axis, respectively. These results are also obtained for the same values of the observer gain $\Gamma$ and the load torque.

Figures 7 and 8 show flux errors in the $(a, b)$ frame, respectively. These results were obtained for a load torque step change illustrated by Figure 9. In this case, Figure 10 shows the rotor speed. These results clearly show that the proposed reduced-order flux estimator is quite robust to external disturbances. Its robustness has also been checked versus parameter variations. Furthermore, it should be mentioned that the same observer gain $\Gamma$ could be adopted for any load torque change.

4.2. Simulation Results of the Reduced-Order Observer for Rotor Fluxes and Speed Estimation. Figures 11 and 12 show the estimated rotor fluxes $\widehat{\varphi}_{a}$ and $\widehat{\varphi}_{b}$, respectively. Figures 1315 illustrate the corresponding real rotor speed, its estimate, and the speed error, respectively. These results were obtained for $\Gamma_{1}=\Gamma_{2}=\Gamma_{3}=\left[\begin{array}{ll}0.8710^{-5} & 10^{-6}\end{array}\right]^{T}$ and a load torque $T_{L}=5 \mathrm{Nm}$.

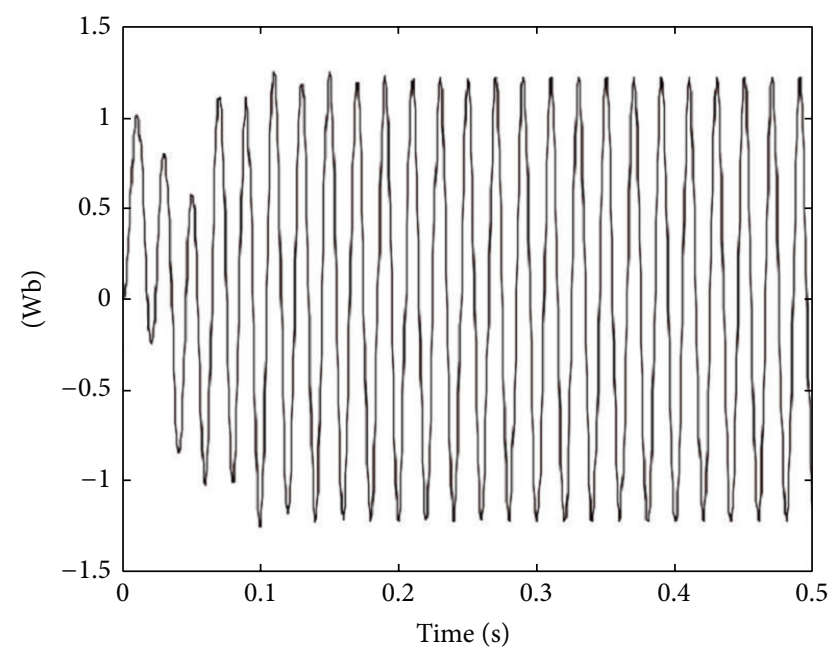

FIgURE 1: Real flux $\varphi_{a}$.

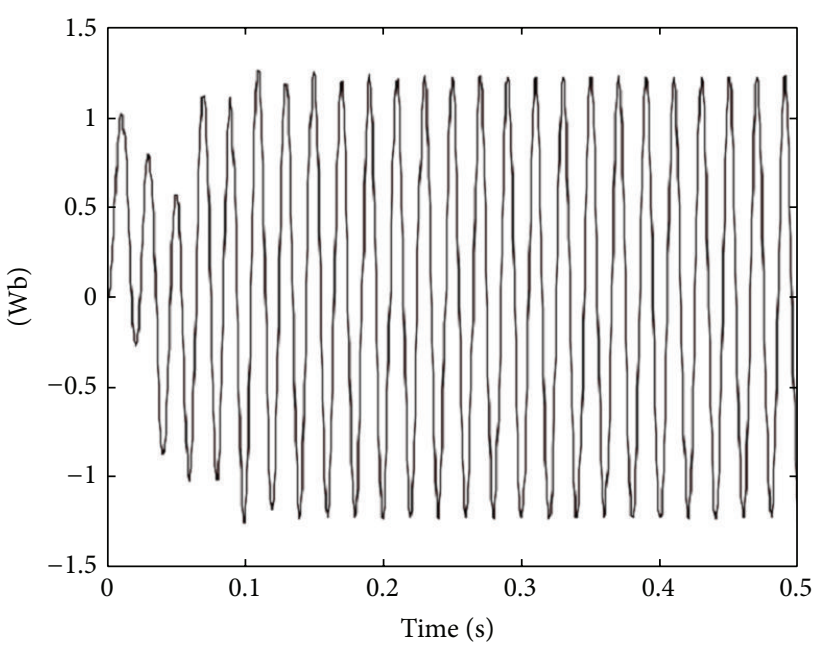

Figure 2: Estimated flux $\widehat{\varphi}_{a}$.

The proposed nonlinear observer obviously gives quite good estimation results. However, in case of load torque variations, the observer gains should be adjusted.

\section{Conclusion}

This paper has investigated the effectiveness of metric observers for induction motor control purposes. Firstly, assuming that stator currents and speed are measured, a metric observer was designed to estimate rotor fluxes. Afterward, assuming that only stator currents are measured, a metric observer was derived to estimate rotor fluxes and speed. The achieved simulation results on a $4 \mathrm{~kW}$ induction motor drive have clearly highlighted the effectiveness of the two proposed observers. Further investigations should be carried out for comparison purposes. 


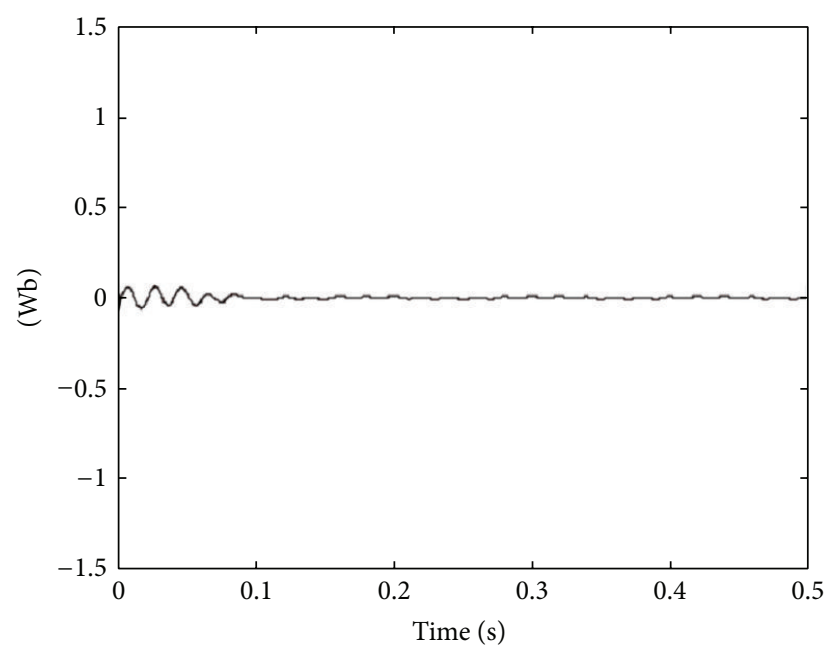

FIgURE 3: Flux error in $a$-axis.

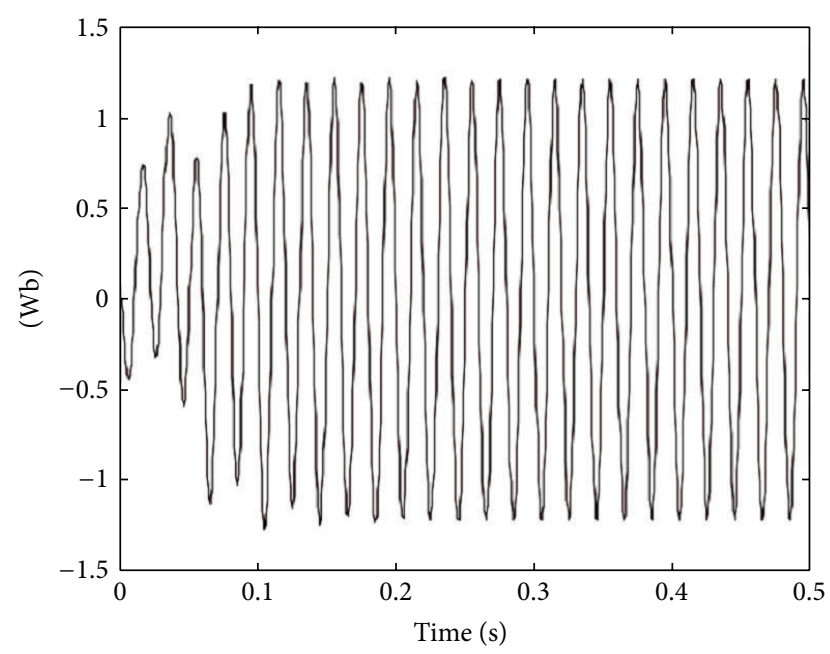

FIgURE 4: Real flux $\varphi_{b}$.

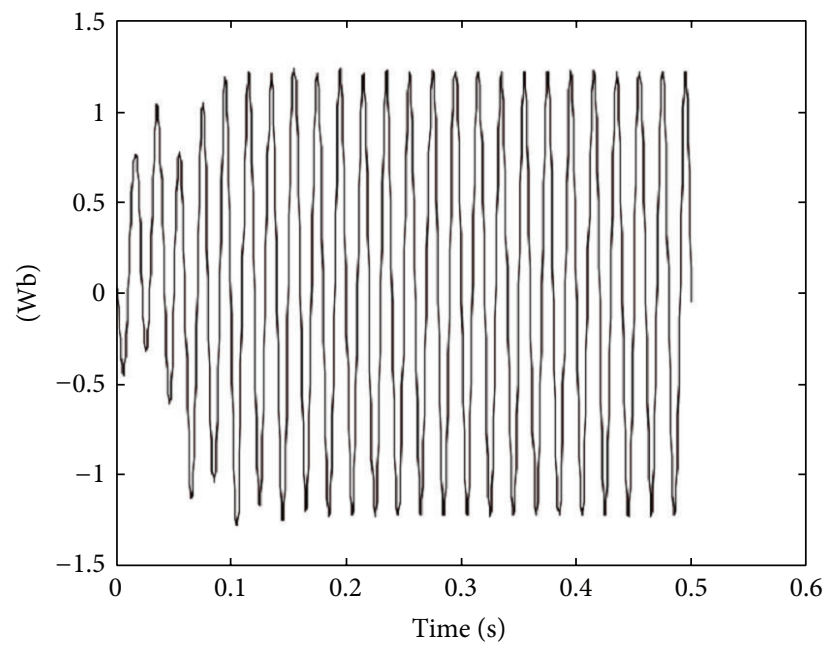

FIgURE 5: Estimated flux $\widehat{\varphi}_{b}$.

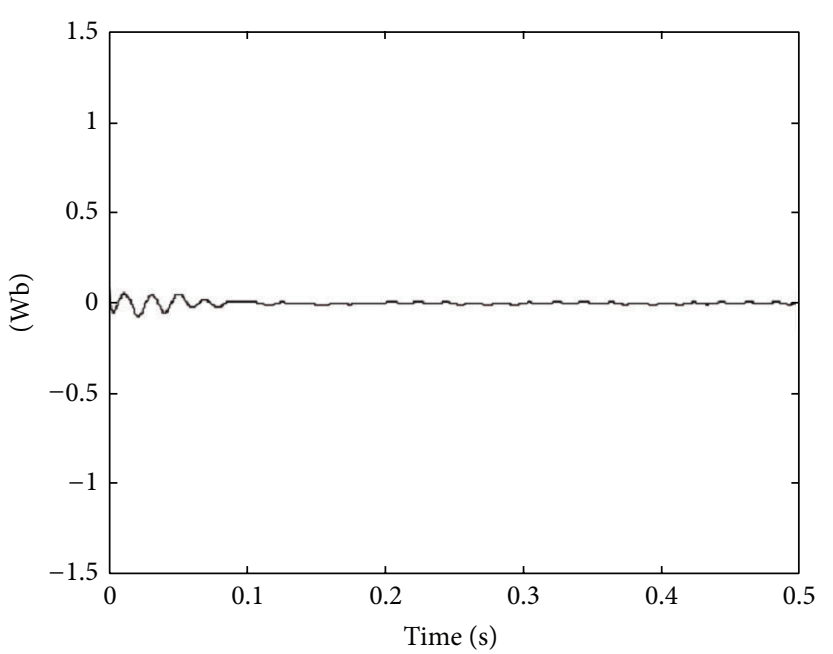

Figure 6: Flux error in $b$-axis.

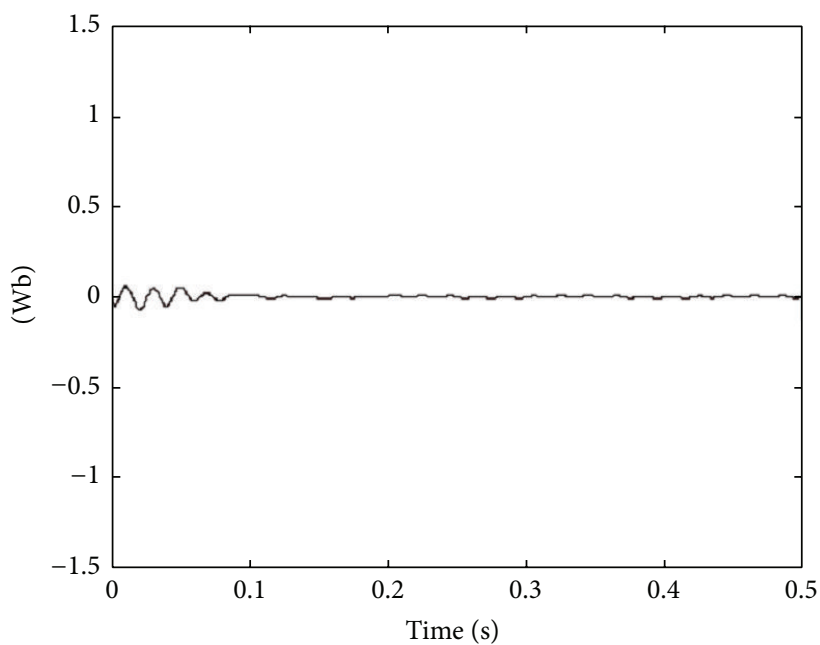

FIgURE 7: Flux estimation error in $a$-axis.

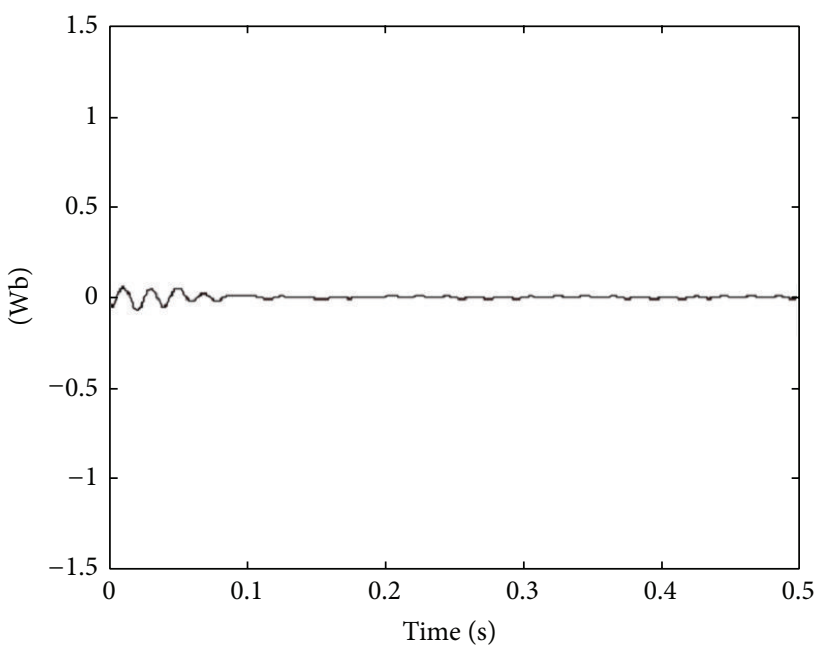

FIgURE 8: Flux estimation error in $b$-axis. 


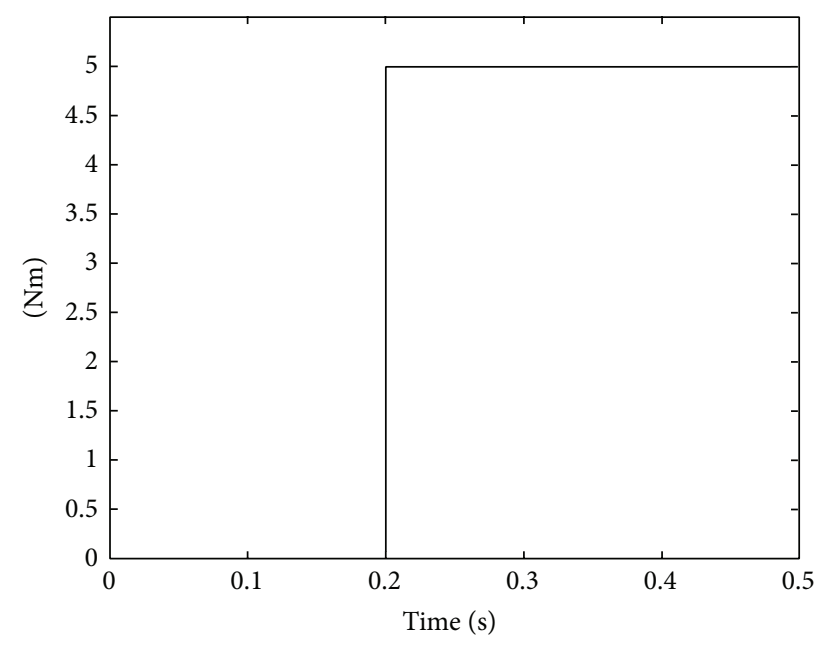

Figure 9: Load torque step change.

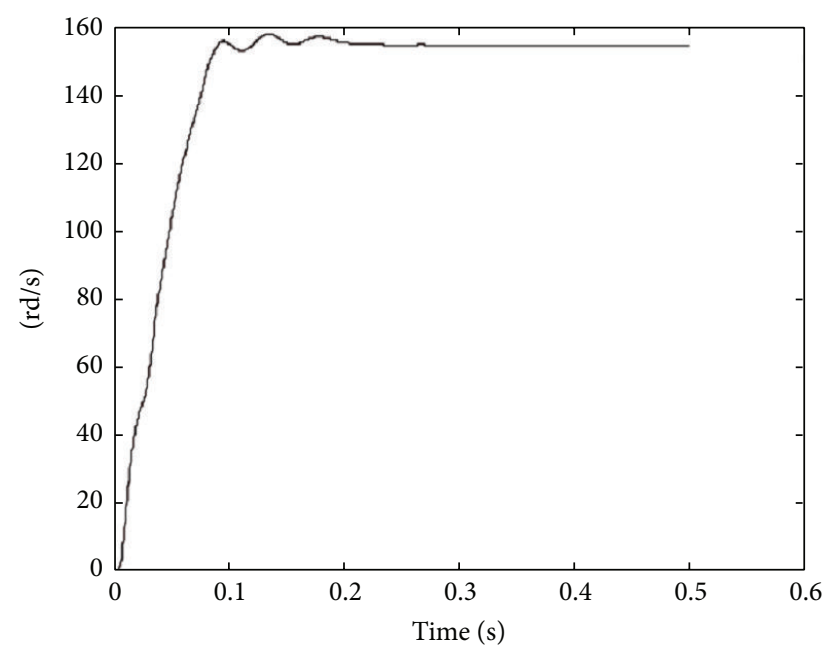

FIGURE 10: Rotor speed.

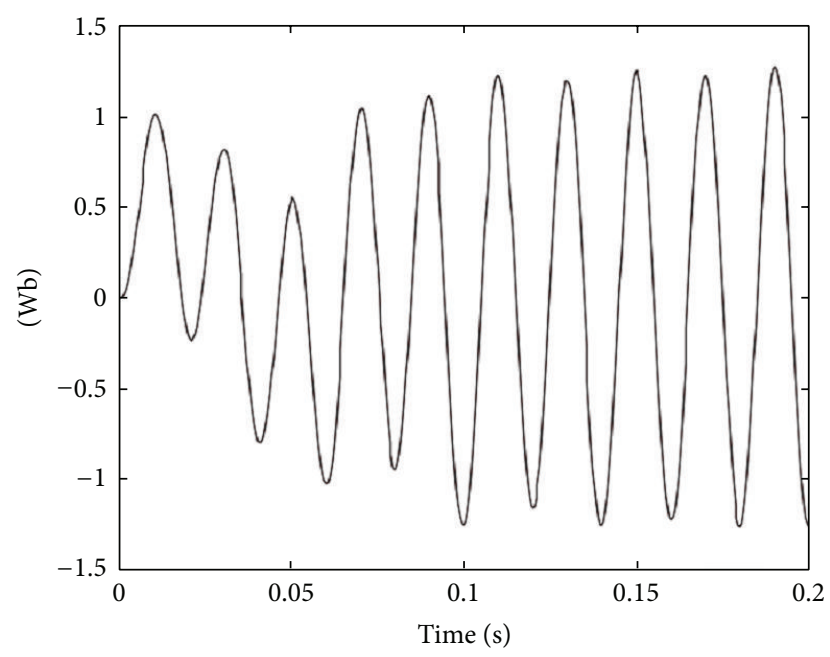

FIGURE 11: Estimated flux $\widehat{\varphi}_{a}$.

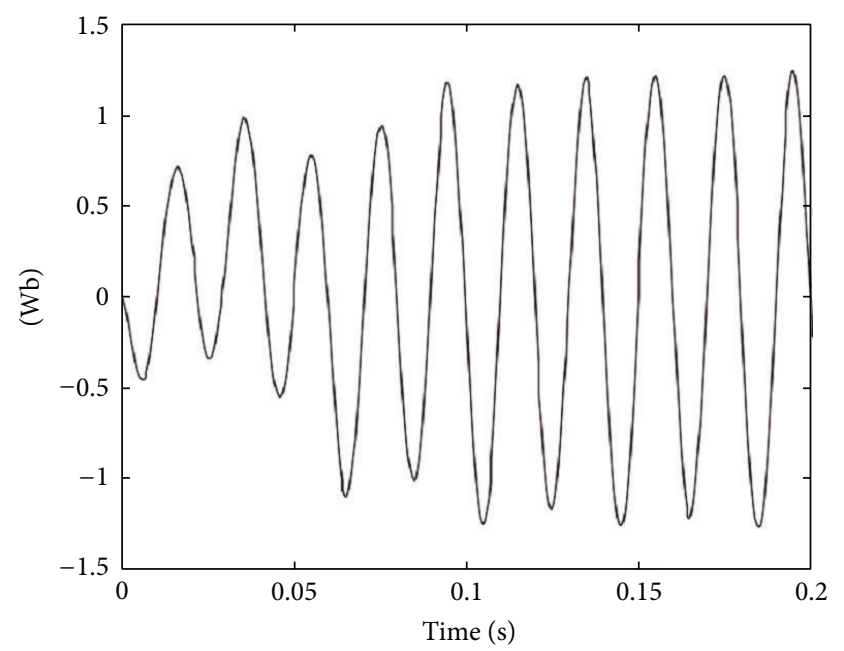

FIgURE 12: Estimated flux $\widehat{\varphi}_{b}$.

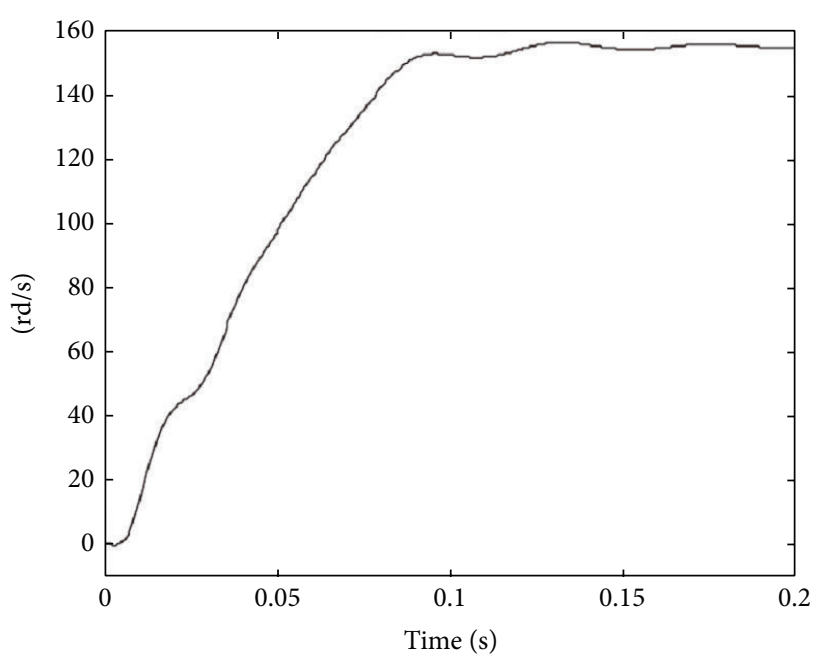

FIgURE 13: Real rotor speed.

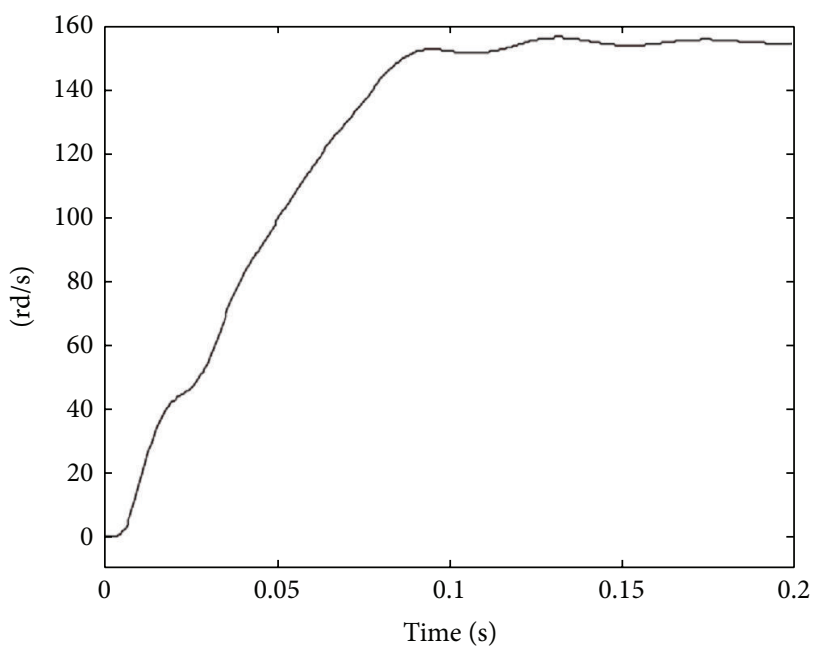

FIGURE 14: Estimated rotor speed. 


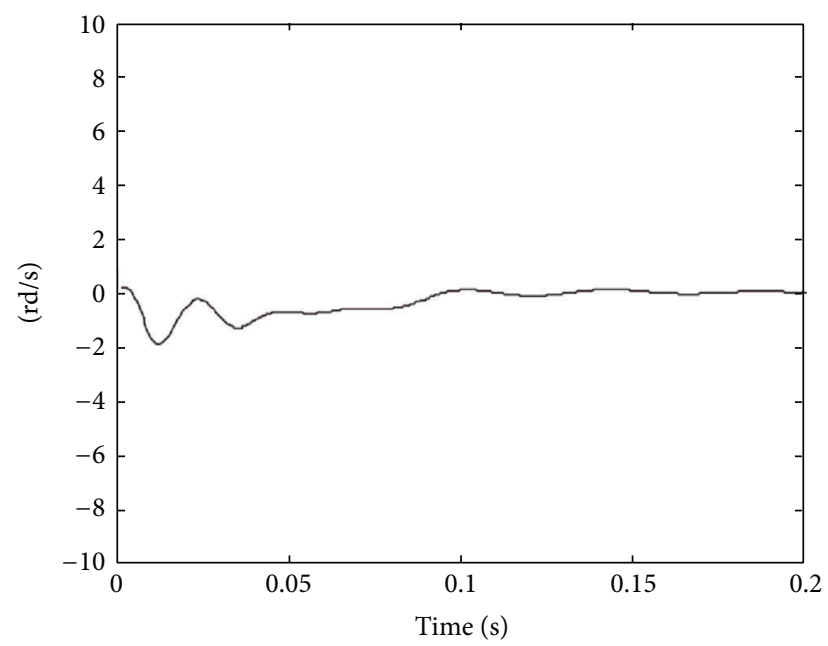

FigURE 15: Rotor speed estimation error.

\section{Nomenclature}

$\begin{array}{ll}i_{a}, i_{b}: & \text { Stator currents } \\ u_{a}, u_{b}: & \text { Stator voltages } \\ \varphi_{a}, \varphi_{b}: & \text { Rotor fluxes } \\ T_{L}: & \text { Load torque } \\ \omega: & \text { Mechanical speed } \\ L_{s}, R_{s}: & \text { Stator inductance and resistance } \\ L_{r}, R_{r}: & \text { Rotor inductance and resistance } \\ M: & \text { Mutual inductance } \\ T_{r}=L_{r} / R_{r}: & \text { Rotor time constant } \\ p: & \text { Pole pairs number } \\ J: & \text { Rotor inertia. }\end{array}$

\section{Competing Interests}

The authors declare that they have no competing interests.

\section{References}

[1] F. Blaschke, "The principle of field orientation as applied to the new transvektor closed-loop control system for rotating field machines," Siemens Review, no. 39, pp. 217-220, 1972.

[2] R. Marino, S. Peresada, and P. Valigi, "Adaptive input-output linearizing control of induction motors," IEEE Transactions on Automatic Control, vol. 38, no. 2, pp. 208-221, 1993.

[3] R. Ortega, C. Canudas, and S. I. Seleme, "Nonlinear control of induction motors: torque tracking with unknown load disturbance," IEEE Transactions on Automatic Control, vol. 38, no. 11, pp. 1675-1680, 1993.

[4] V. I. Utkin, "Sliding mode control design principles and applications to electric drives," IEEE Transactions on Industrial Electronics, vol. 40, no. 1, pp. 23-36, 1993.

[5] B. Beltran, M. E. H. Benbouzid, and T. Ahmed-Ali, "Secondorder sliding mode control of a doubly fed induction generator driven wind turbine," IEEE Transactions on Energy Conversion, vol. 27, no. 2, pp. 261-269, 2012.

[6] A. Benchaib, A. Rachid, E. Audrezet, and M. Tadjine, "Real-time sliding-mode observer and control of an induction motor," IEEE
Transactions on Industrial Electronics, vol. 46, no. 1, pp. 128-138, 1999.

[7] A. Benchaib, A. Rachid, and E. Audrezet, "Sliding mode inputoutput linearization and field orientation for real-time control of induction motors," IEEE Transactions on Power Electronics, vol. 14, no. 1, pp. 3-13, 1999.

[8] M. Benbouzid, "High-order sliding mode control of DFIGbased wind turbines," in Wind Turbine Control and Monitoring, N. Luo, Y. Vidal, and L. Acho, Eds., Advances in Industrial Control, chapter 2, pp. 23-48, Springer, Berlin, Germany, 2014.

[9] B. Beltran, T. Ahmed-Ali, and M. E. H. Benbouzid, "High-order sliding-mode control of variable-speed wind turbines," IEEE Transactions on Industrial Electronics, vol. 56, no. 9, pp. 33143321, 2009.

[10] J.-F. Stumper, V. Hagenmeyer, S. Kuehl, and R. Kennel, "Deadbeat control for electrical drives: a robust and performant design based on differential flatness," IEEE Transactions on Power Electronics, vol. 30, no. 8, pp. 4585-4596, 2015.

[11] C. Schauder, "Adaptive speed identification for vector control of induction motors without rotational transducers," IEEE Transactions on Industry Applications, vol. 28, no. 5, pp. 10541061, 1992.

[12] H. Kubota, K. Matsuse, and T. Nakano, "DSP-based speed adaptive flux observer of induction motor," IEEE Transactions on Industry Applications, vol. 29, no. 2, pp. 344-348, 1993.

[13] M. E. H. Benbouzid, B. Beltran, Y. Amirat, and S. Breton, "Sensorless control of doubly-fed induction generator-based wind turbines using a high-order sliding mode observer," International Review of Electrical Engineering, vol. 9, no. 1, pp. 49-55, 2014.

[14] B. Beltran, M. E. H. Benbouzid, T. Ahmed-Ali, and H. Mangel, "DFIG-based wind turbine robust control using high-order sliding modes and a high gain observer," International Review on Modelling and Simulations, vol. 4, no. 3, pp. 1148-1155, 2011.

[15] M. Y. Hammoudi, A. Allag, M. Becherif, M. E. H. Benbouzid, and H. Alloui, "Observer design for induction motor: an approach based on the mean value theorem," Frontiers in Energy, vol. 8, no. 4, pp. 426-433, 2014.

[16] W. Lohmiller and J. J. E. Slotine, "On metric controllers and observers for nonlinear systems," in Proceedings 35th IEEE Conference on Decision and Control (CDC '96), vol. 2, pp. 14771482, Kobe, Japan, December 1996.

[17] W. Lohmiller and J.-J. E. Slotine, "Applications of metric observers for nonlinear systems," in Proceedings of the IEEE International Conference on Control Applications (CCA '96), pp. 367-372, Dearborn, Mich, USA, September 1996. 


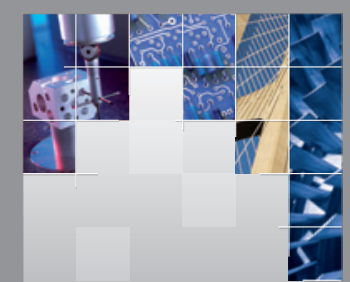

\section{Enfincering}
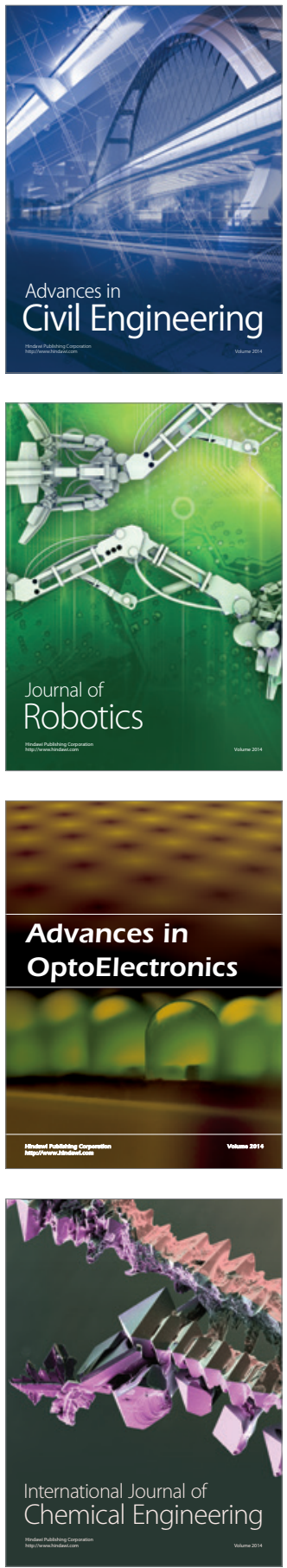

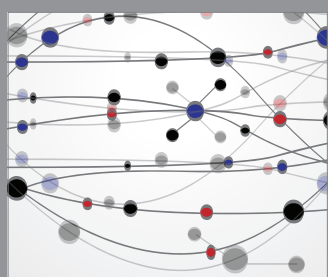

The Scientific World Journal

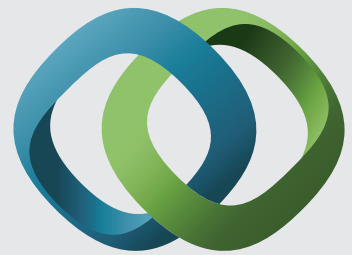

\section{Hindawi}

Submit your manuscripts at

http://www.hindawi.com
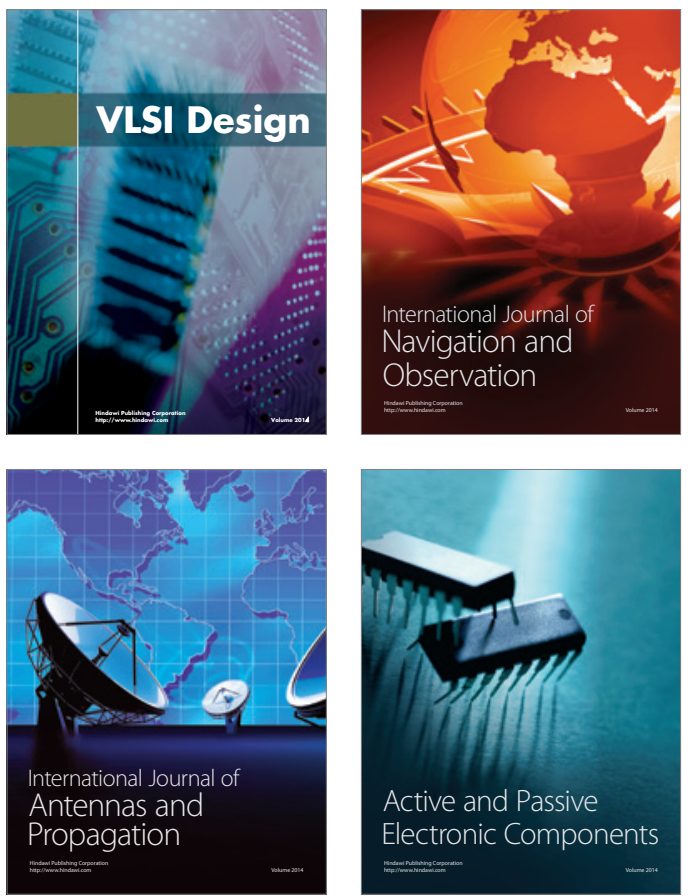
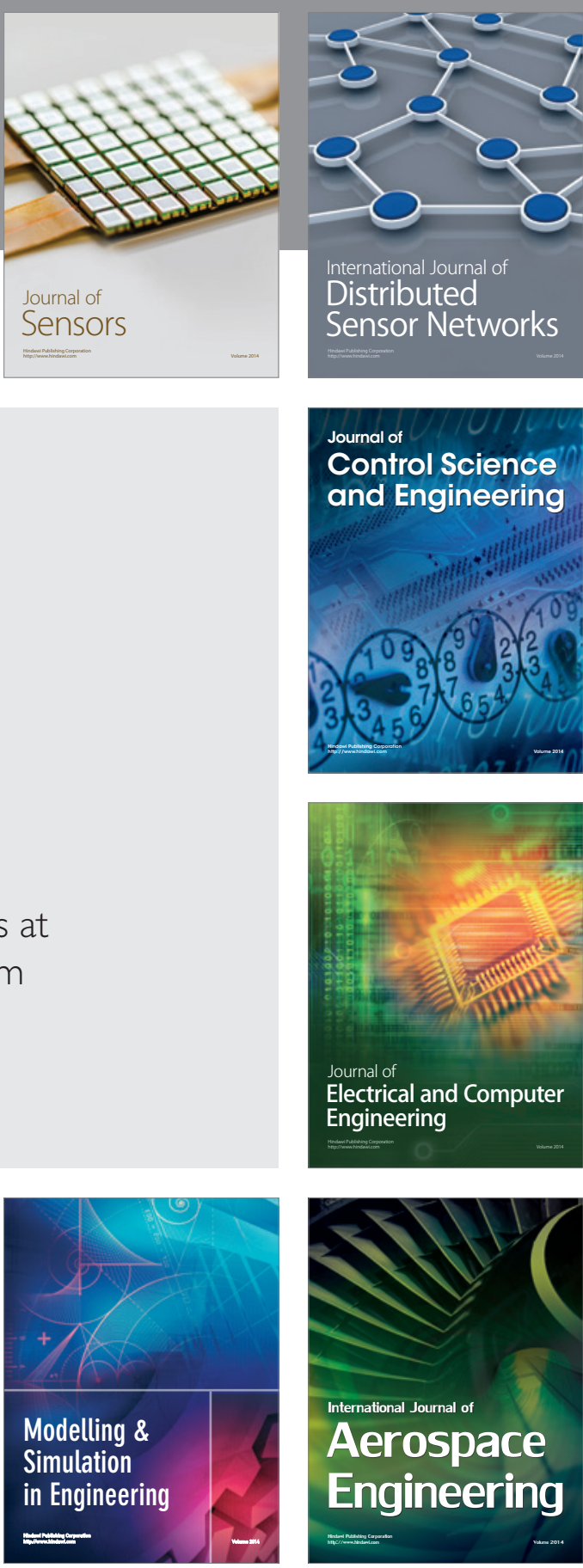

International Journal of

Distributed

Sensor Networks

Journal of

Control Science

and Engineering
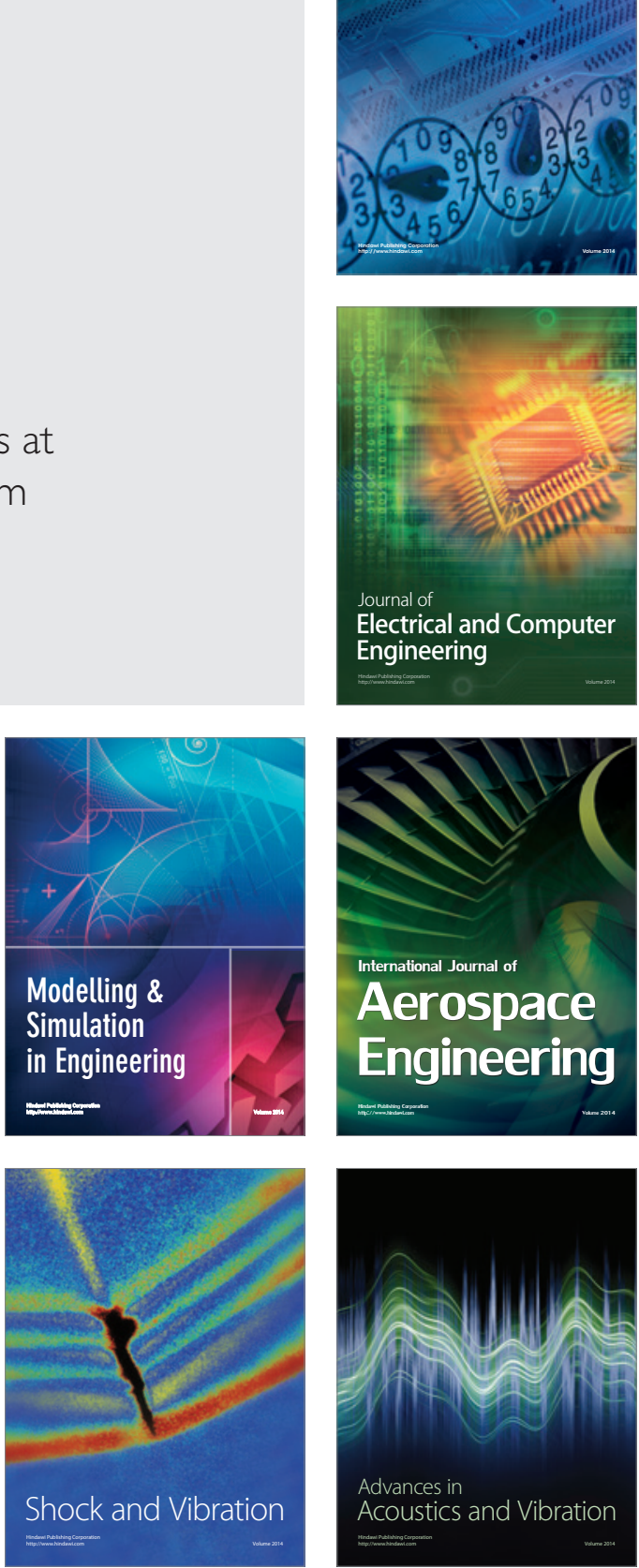